\title{
How should we model irreversible reactions in Earth surface processes?
}

\author{
CHEN ZHU ${ }^{1}$ \\ ${ }^{1}$ Department of Earth and Atmospheric Sciences, Indiana \\ University, Bloomington, IN 47405, USA
}

In low-temperature Earth surface processes such as weathering, reactions in groundwater aquifers, and geological carbon sequestration, the reverse reaction of dissolution, namely, precipitation may not occur significantly for most silicates in the near-equilibrium but undersaturated solutions. Conventionally, geochemists have dealt with net reactions, not unidireactional reactions. Let's use feldspar as an example. Consider that there is a unidirectional dissolution reaction. Approaching equilibrium but still undersaturated, the reverse reaction of unidirectional feldspar precipitation reaction becomes more important. Feldspar continues to (net) dissolve from far from equilibrium to near-equilibrium till reaching equilibrium. The net dissolution rate is the sum of unidirectional dissolution and precipitation rates and this is accounted by including an affinity term in the rate law. The rate laws currently used in coupled reactive transport models reflect this thinking, but carry a significant and often unnoticed assumption that treats feldspar weathering as a reversible reaction in low-temperature Earth surface processes.

The isotope tracer method of geochemical kinetics studies now allows us to separate the unidirectional dissolution and precipitation reaction rates. Recent experiments demonstrate that feldspar precipitation in near-equilibrium but undersaturated solutions were negligible at $50{ }^{\circ} \mathrm{C}$ [1], consistent with the analysis of sedimentary records [2]. In light of these new data, we recommend using the unidirectional dissolution rate constants without an affinity term together with the precipitation of secondary phases such as kaolinite from supersaturation.

Even without an affinity term for feldspar, the system of coupled dissolution and precipitation reactions will not go to equilibrium with respect to feldspars. Instead, the system will stay close to equilibrium. Feldspar will continue to dissolve, and the secondary phase continues to precipitate until all feldspars in the system are exhausted.

[1] Zhu C, Rimstidt JD, Zhang YL, Kang JT, Yuan HL (2020). Geochimica et Cosmochimica Acta. v271, 132-153. DOI: 10.1016/j.gca.2019.12.024. [2] Kastner, M. and Siever, R. (1979) Low temperature feldspars in sedimentary rocks. American Journal of Science 279, 435-479. 